\title{
Rotation-Translation Coupling Analysis on Perturbed Spacecraft Relative Translational Motion
}

\author{
Jianlin Chen • Josep J. Masdemont • Gerard Gómez • Jianping Yuan · \\ Zhanxia Zhu
}

Received: date / Accepted: date

\begin{abstract}
This paper explores an accurate and complete spacecraft six-degrees-of-freedom coupled relative motion model using the dual quaternion representation. Based on this technique, we build a scheme capable of describing both kinematic and dynamic coupling effects on the spacecraft relative translational motion through a further combination with the chaser's precise absolute translational and rotational dynamics. This new model generalizes the existing nonlinear spacecraft relative translational model to include both the kinematic coupling effect due to the displacements of selected feature points relative to the spacecraft centers of mass and the dynamic coupling effect induced by the gravity gradient torque and the orbital perturbations. Several numerical simulations are implemented to validate the
\end{abstract}

\section{Jianlin Chen}

National Key Laboratory of Aerospace Flight Dynamics, School of Astronautics, Northwestern Polytechnical University, Xi'an, Shaanxi, 710072, China; IEEC \& Departament de Matemàtiques, Universitat Politècnica de Catalunya, Diagonal 647, 08028 Barcelona.

E-mail: chenjl@mail.nwpu.edu.cn

Josep J. Masdemont

IEEC \& Departament de Matemàtiques, Universitat Politècnica de Catalunya, Diagonal 647, 08028 Barcelona.

Gerard Gómez

IEEC \& Departament de Matemàtiques i Informàtica, Universitat de Barcelona, Gran Via de les Corts Catalanes 585, 08007 Barcelona.

Jianping Yuan

National Key Laboratory of Aerospace Flight Dynamics, School of Astronautics, Northwestern Polytechnical University, Xi'an, Shaanxi, 710072, China.

Zhanxia Zhu

National Key Laboratory of Aerospace Flight Dynamics, School of Astronautics, Northwestern Polytechnical University, Xi'an, Shaanxi, 710072, China. feasibility of the proposed model, for analyzing both the kinematic and dynamic coupling effects, on the relative translational motion of two arbitrary feature points in either Keplerian or $J_{2}$ perturbed orbits. The results are further compared against the $J_{2}$ perturbation effect on the relative translational motion.

Keywords Dual quaternions - Relative motion · Dynamic coupling · Kinematic coupling · Orbital perturbation

\section{Introduction}

The precise prediction and capture of the relative motion between arbitrary feature points on two tumbling spacecraft are fundamental technologies required for the construction of autonomous distributed space systems, spacecraft formation flying (SFF) $[1,2]$, close rendezvous, and docking (R\&D) [3-6]. Growing research interest in such space missions, consisting of multiple spacecraft, motivates the development of an accurate modeling on relative spacecraft position and orientation.

The common three-degrees-of-freedom (DOF) pointmass model was firstly proposed to study the relative translational motion of two spacecraft. A well known reduced model referred as the Clohessy-Wiltshire equations was proposed under the assumption of a circular reference trajectory and a spherical Earth [7]. Further studies were carried out by means of generalizing circular reference trajectories to elliptic ones [8-11] or considering multiple perturbations, such as drag [12] and an oblate Earth $[1,13]$. However the aforementioned models can only be employed to predict relative translational motion between two spacecraft centers of mass 
$(\mathrm{CMs})$, this is, the spacecraft relative rotation is omitted.

In general, the six-DOF coupled relative motion between two rigid spacecraft can be formulated using different representations, i.e. the Lie group $\operatorname{SE}(3)[3,14]$, dual quaternions $[15,16]$, or Euler angles and translation terms. Based on geometric mechanics (i.e. the nonlinear manifold $\mathrm{SE}(3)$ ), the relative rotational and translational motion can be represented in a coupled and singularity-free way, such that one unified control law is designed to solve the six-DOF relative motion control problem [3]. Another widely used technique for addressing the coupled relative motion of rigid spacecraft without singularities is the dual quaternion parametrization, which provides a unified compact form of the dynamics $[15,17]$. Similar to the usage of the nonlinear manifold SE(3), dual quaternion representation simplifies the controller development by allowing one unified control law to be obtained for coupled spacecraft relative motions $[16,17]$. In particular, it is relatively simple to extend existing attitude controllers based on quaternions into the coupled position and attitude controllers based on dual quaternions, and with analogous stability properties $[17,19]$. Besides, it has been shown that dual quaternions possess higher computational efficiency and lower storage cost than other commonly used representations [20,21]. On the downside, the coupled relative motion model formulated on the dual quaternion representation encounters the so-called unwinding phenomenon, which inherits from the classical quaternions. Solutions for this issue have been studied in $[16,17]$.

Note that a high-precision description of relative rotational and translational motion needs to consider two coupling effects, including both the internal kinematic and the external dynamic couplings. Kinematic coupling essentially can be seen as a projection of the rotational motion about the $\mathrm{CM}$ on the relative translational configuration space $[5,6]$. Clearly, it is induced by the motion itself rather than from external perturbations. Dynamic coupling effect stemming from all the external perturbations (such as Earth's non-spherical gravity [22], solar radiation pressure [23,24], atmospheric drag [25] or control force [15]) depends on the spacecraft attitude or position, and affects both rotational and translational dynamics. The models for the dominant external forces and torques governing the spacecraft's absolute translational and rotational motion can be found in $[26,27]$.

In the previous works, the authors do not address displacements of non-CM feature points to explore the kinematic coupling effect, which only arises when one considers the relative motion between non-CM points. In order to analyze this kinematic coupling on the space- craft relative translation, Segal and Gurfil [5] proposed a six-DOF relative dynamic model between two rigid spacecraft under the assumption that the chaser bodyfixed frame is aligned with the reference orbital frame. Lee and Vukovich [6] presented another kinematically coupled relative spacecraft motion model about two arbitrary feature points, on the chief and deputy spacecraft, by mitigating the attitude synchronization assumption, this is, the restrictive alignment of the bodyfixed and orbital frames. These relative motion models were obtained in the orbital reference frame, with the assumption of Keplerian elliptical nominal orbits, and do not consider the analysis of the rotation-translation coupling effect on spacecraft relative translation in a perturbed orbit environment.

The present study is devoted to studying the kinematic and dynamic coupling effects on the relative translational motion in a general orbital case. We propose a six-DOF coupled relative motion model to describe the relative position of two arbitrary feature points on the target and chaser spacecraft, without the Keplerian reference orbit assumption. In addition, the proposed model enables to analyze $J_{2}$ perturbation effects. Therefore, the magnitudes of the kinematic and dynamic coupling effects and of the perturbation effect on the relative translational motion can be analyzed.

The reminder of the paper is organized as follows. The mathematical preliminaries used throughout the whole paper are introduced in Section 2. Section 3 addresses the formulation of the relative motion of two arbitrary non-CM points on two close spacecraft using a dual quaternion representation. Section 4 provides some illustrative simulations to validate the effectiveness of the proposed model. This includes the implementation of several numerical simulations that analyze the kinematic and dynamic coupling effects on the relative translational motion: on an ideal Keplerian reference orbit or on a $J_{2}$ perturbed reference orbit. Finally, the conclusions are provided in Section 5.

\section{Mathematical preliminaries}

The purpose of this section is to provide a compact and concise summary of quaternions and dual quaternions, as well as the basic operational rules adopted in this paper. For more details, the reader can refer to $[2,17$, 28-31].

\subsection{Quaternions}

Quaternions were invented by Sir William Rowan Hamilton in 1843 [32]. They are generally employed for the 
rotation analysis of a rigid spacecraft without singularities [33].

A quaternion is defined as an ordered pair $\left(q_{0}, \overline{\boldsymbol{q}}\right)$,

$\boldsymbol{q}=q_{0}+q_{1} \boldsymbol{i}+q_{2} \boldsymbol{j}+q_{3} \boldsymbol{k}$,

where $q_{0}$ is the scalar part, and $\overline{\boldsymbol{q}}=q_{1} \boldsymbol{i}+q_{2} \boldsymbol{j}+q_{3} \boldsymbol{k}$ is the vector part. If $q_{0}=0, \boldsymbol{q}$ is called a vector quaternion, which is always exploited to describe the translation of a spacecraft. Table 1 provides a summary of basic algebraic operations on quaternions. More information can be found in [17].

Table 1 Algebra of quaternions

\begin{tabular}{cc}
\hline Operation & Formulae \\
\hline Conjugation & $\boldsymbol{q}^{*}=\left(q_{0},-\overline{\boldsymbol{q}}\right)$ \\
Addition & $\boldsymbol{q}+\lambda \boldsymbol{p}=\left(q_{0}+\lambda p_{0}, \overline{\boldsymbol{q}}+\lambda \overline{\boldsymbol{p}}\right), \lambda \in \mathbb{R}$ \\
Multiplication & $\boldsymbol{q} \circ \boldsymbol{p}=\left(q_{0} p_{0}-\overline{\boldsymbol{q}} \cdot \overline{\boldsymbol{p}}, q_{0} \overline{\boldsymbol{p}}+p_{0} \overline{\boldsymbol{q}}+\overline{\boldsymbol{q}} \times \overline{\boldsymbol{p}}\right)$ \\
Dot product & $\boldsymbol{q} \cdot \boldsymbol{p}=\left(q_{0} p_{0}+\overline{\boldsymbol{q}} \cdot \overline{\boldsymbol{p}}, \overline{\mathbf{0}}\right)$ \\
Cross product & $\boldsymbol{q} \times \boldsymbol{p}=\left(0, q_{0} \overline{\boldsymbol{p}}+p_{0} \overline{\boldsymbol{q}}+\overline{\boldsymbol{q}} \times \overline{\boldsymbol{p}}\right)$ \\
\hline
\end{tabular}

Note: if $\boldsymbol{q}$ and $\boldsymbol{p}$ are vector quaternions, $\boldsymbol{q} \times \boldsymbol{p}=\frac{1}{2}(\boldsymbol{q} \circ \boldsymbol{p}-\boldsymbol{p} \circ \boldsymbol{q})$.

Note that the formulae $\overline{\boldsymbol{q}} \cdot \overline{\boldsymbol{p}}$ and $\overline{\boldsymbol{q}} \times \overline{\boldsymbol{p}}$ in Table 1 have to be formally understood as the classical dot and cross products for vectors $\overline{\boldsymbol{q}}$ and $\overline{\boldsymbol{p}}$ in $\mathbb{R}^{3}$. In addition, since $\overline{\boldsymbol{v}}$ is a vector in $\mathbb{R}^{3}$, it is embedded in the quaternion algebra considering the vector quaternion $\boldsymbol{v}=(0, \overline{\boldsymbol{v}})$, thus an expression $\boldsymbol{q}^{*} \circ \overline{\boldsymbol{v}} \circ \boldsymbol{q}$ should be understood as $\boldsymbol{q}^{*} \circ \boldsymbol{v} \circ \boldsymbol{q}$.

On the other hand, $\boldsymbol{q}$ is referred to as a unit quaternion if $\boldsymbol{q}^{*} \circ \boldsymbol{q}=\boldsymbol{q} \circ \boldsymbol{q}^{*}=1$. A unit quaternion is,

$\boldsymbol{q}=\left(q_{0}, \overline{\boldsymbol{q}}\right)=\left(\cos \frac{\theta}{2}, \sin \frac{\theta}{2} \boldsymbol{n}\right)$,

for a certain angle $\theta$ and a unit vector $\boldsymbol{n} \in \mathbb{R}^{3}$. Clearly it describes the attitude of a rigid spacecraft by means of the rotation angle $\theta$ about the $\boldsymbol{n}$ axis (see [28]). However, on the bad side, it is also worth to remind here the existence of the unwinding phenomenon when quaternions are employed for the attitude description of a rigid spacecraft and the design of control laws [31,34].

\subsection{Dual quaternions}

Let us consider the symbol $\varepsilon$, known as the dual unit. It has the property that formally satisfies $\varepsilon^{2}=0$ (it is nilpotent) and commutes with every element of the algebra. With this concept, dual vectors and dual quaternions were introduced by Clifford [30] as follows:

Dual vector (or "motor"):

$\hat{\boldsymbol{a}}=\boldsymbol{a}_{r}+\varepsilon \boldsymbol{a}_{d}$, with $\boldsymbol{a}_{r}, \boldsymbol{a}_{d}$ vectors in $\mathbb{R}^{3}$,
Dual quaternion:

$\hat{\boldsymbol{q}}=\boldsymbol{q}_{r}+\varepsilon \boldsymbol{q}_{d}$, with $\boldsymbol{q}_{r}, \boldsymbol{q}_{d}$ real quaternions,

where the subscripts " $r$ " and " $d$ " in the previous expressions stand for the so called real and dual parts of the dual element respectively. Similar to the standard quaternion case, we also define two specific sets of dual quaternions:

Dual vector quaternion:

$\hat{\boldsymbol{q}}=\boldsymbol{q}_{r}+\varepsilon \boldsymbol{q}_{d}$, with, $q_{r 0}=q_{d 0}=0$.

Dual unit quaternion:

$\hat{\boldsymbol{q}}=\boldsymbol{q}_{r}+\varepsilon \boldsymbol{q}_{d}$, with, $\hat{\boldsymbol{q}}^{*} \circ \hat{\boldsymbol{q}}=\hat{\boldsymbol{q}} \circ \hat{\boldsymbol{q}}^{*}=\mathbf{1}$,

where the conjugate $\hat{\boldsymbol{q}}^{*}$ of a dual quaternion and the product of "o" between two dual quaternions are defined in Table 2. Besides, for the convenience of the reader, this table also illustrates some basic algebraic rules on dual quaternions. More details and applications can be found in $[31,35,36]$.

Table 2 Algebra of dual quaternions

\begin{tabular}{cc}
\hline Operation & Formulae \\
\hline Conjugation & $\hat{\boldsymbol{q}}^{*}=\boldsymbol{q}_{r}^{*}+\varepsilon \boldsymbol{q}_{d}^{*}$ \\
Addition & $\hat{\boldsymbol{q}}+\lambda \hat{\boldsymbol{p}}=\left(\boldsymbol{q}_{r}+\lambda \boldsymbol{p}_{r}\right)+\varepsilon\left(\boldsymbol{q}_{d}+\lambda \boldsymbol{p}_{d}\right), \lambda \in \mathbb{R}$ \\
Multiplication & $\hat{\boldsymbol{q}} \circ \hat{\boldsymbol{p}}=\left(\boldsymbol{q}_{r} \circ \boldsymbol{p}_{r}\right)+\varepsilon\left(\boldsymbol{q}_{r} \circ \boldsymbol{p}_{d}+\boldsymbol{q}_{d} \circ \boldsymbol{p}_{r}\right)$ \\
Dot product & $\hat{\boldsymbol{q}} \cdot \hat{\boldsymbol{p}}=\left(\boldsymbol{q}_{r} \cdot \boldsymbol{p}_{r}\right)+\varepsilon\left(\boldsymbol{q}_{r} \cdot \boldsymbol{p}_{d}+\boldsymbol{q}_{d} \cdot \boldsymbol{p}_{r}\right)$ \\
Cross product & $\hat{\boldsymbol{q}} \times \hat{\boldsymbol{p}}=\left(\boldsymbol{q}_{r} \times \boldsymbol{p}_{r}\right)+\varepsilon\left(\boldsymbol{q}_{r} \times \boldsymbol{p}_{d}+\boldsymbol{q}_{d} \times \boldsymbol{p}_{r}\right)$ \\
\hline
\end{tabular}

Note: if $\hat{\boldsymbol{q}}$ and $\hat{\boldsymbol{p}}$ are dual vector quaternions, $\hat{\boldsymbol{q}} \times \hat{\boldsymbol{p}}=\frac{1}{2}(\hat{\boldsymbol{q}} \circ \hat{\boldsymbol{p}}-$ $\hat{\boldsymbol{p}} \circ \hat{\boldsymbol{q}})$.

Analogously, a dual vector $\hat{\boldsymbol{a}}$ is embedded in the dual quaternion algebra considering $\hat{\boldsymbol{v}}=\left(0, \boldsymbol{a}_{r}\right)+\varepsilon\left(0, \boldsymbol{a}_{d}\right)$. Expressions of the form $\hat{\boldsymbol{q}}^{*} \circ \hat{\boldsymbol{a}} \circ \hat{\boldsymbol{q}}$ have to be understood as $\hat{\boldsymbol{q}}^{*} \circ \hat{\boldsymbol{v}} \circ \hat{\boldsymbol{q}}$. In addition, the symbol $\frac{d}{d \varepsilon}$ can be also seen as an operator that formally acts on dual elements,

$\frac{d}{d \varepsilon} \hat{\boldsymbol{q}}=\frac{d}{d \varepsilon}\left(\boldsymbol{q}_{r}+\varepsilon \boldsymbol{q}_{d}\right)=\boldsymbol{q}_{d}$,

where we assume that $\frac{d}{d \varepsilon}$ commutes with $\varepsilon$, that is $\varepsilon \frac{d}{d \varepsilon}=\frac{d}{d \varepsilon} \varepsilon=1$, and also, formally, we have $\frac{d^{2}}{d \varepsilon^{2}}=$ $\frac{d}{d \varepsilon} \frac{d}{d \varepsilon} \equiv 0$.

Finally, let us introduce dual matrices. A dual matrix is an element of the form,

$$
\hat{\boldsymbol{M}}=M_{r}+M_{d} \varepsilon+M_{c} \frac{d}{d \varepsilon},
$$

where $M_{r}, M_{d}$ and $M_{c}$ are $3 \times 3$ real matrices. The action of $\hat{\boldsymbol{M}}$ on a dual vector, $\hat{\boldsymbol{v}}=\boldsymbol{v}_{r}+\varepsilon \boldsymbol{v}_{d}$, gives the dual vector,

$$
\hat{\boldsymbol{M}} \hat{\boldsymbol{v}}=\left(M_{r} \boldsymbol{v}_{r}+M_{c} \boldsymbol{v}_{d}\right)+\varepsilon\left(M_{r} \boldsymbol{v}_{d}+M_{d} \boldsymbol{v}_{r}\right) .
$$


It is worth to remark that if we formally multiply two dual matrices, $\hat{\boldsymbol{M}}^{1}$ and $\hat{\boldsymbol{M}}^{2}$, the result is a dual matrix, but in general, $\left(\hat{\boldsymbol{M}}^{1} \hat{\boldsymbol{M}}^{2}\right) \hat{\boldsymbol{v}} \neq \hat{\boldsymbol{M}}^{1}\left(\hat{\boldsymbol{M}}^{2} \hat{\boldsymbol{v}}\right)$, as it can be easily checked. This fact has implications in the definition of the inverse of a dual matrix. When it exists, the inverse of a dual matrix $\hat{\boldsymbol{M}}$ will be a dual matrix $\hat{\boldsymbol{M}}^{-1}$ such that $\hat{\boldsymbol{M}}^{-1}(\hat{\boldsymbol{M}} \hat{\boldsymbol{v}})=\hat{\boldsymbol{M}}\left(\hat{\boldsymbol{M}}^{-1} \hat{\boldsymbol{v}}\right)=\hat{\boldsymbol{v}}$, but this does not mean that the formal product we obtained for $\hat{\boldsymbol{M}}^{-1} \hat{\boldsymbol{M}}$ would be $\boldsymbol{I}=\operatorname{diag}(1,1,1)$.

In this paper the dual inertia matrix is used to describe the main features of the spacecraft such as its mass and inertia matrix, while forces and torques will be represented in a compact way by means of dual vectors. The action of the dual matrix on the dual vector is a concise and simple way to account for both translational and rotational dynamics of a spacecraft.

\section{Problem formulation}

In a close SFF configuration orbiting the Earth one spacecraft is referred to as the chaser and the other one as the target. Consider the relative motion of two arbitrary points on the chaser and target, we first derive the dynamically coupled relative motion model under the external perturbing forces and torques. Then, the sixDOF coupled model describing the relative motion between two non-CMs feature points is further proposed. In the development of these models, we need of three coordinate reference systems defined as shown in Fig. 1. The Earth-centered inertial (ECI) frame $\mathcal{I}$ is a standard Cartesian right-hand reference frame attached to the Earth's center of mass. The chaser body-fixed frame $\mathcal{C}$ is aligned with the principal axis of inertia of the chaser and attached to its center of mass. The target body-fixed frame $\mathcal{T}$ is similarly defined as $\mathcal{C}$ but on the target spacecraft.

\subsection{Dynamically coupled relative motion model}

Using the dual quaternion representation, the kinematic equation of a rigid chaser is governed by[29],

$\dot{\hat{\boldsymbol{q}}}_{c}=\frac{1}{2} \hat{\boldsymbol{q}}_{c} \circ^{\mathcal{C}} \hat{\boldsymbol{\omega}}_{c}$,

where $\hat{\boldsymbol{q}}_{c}$ denotes the chaser's position and orientation in the inertial frame $\mathcal{I},{ }^{\mathcal{C}} \hat{\boldsymbol{\omega}}_{c}$ indicates the chaser's velocity motor expressed in the body-fixed frame $\mathcal{C}$ and,

${ }^{\mathcal{C}} \hat{\boldsymbol{\omega}}_{c}={ }^{\mathcal{C}} \boldsymbol{\omega}_{c}+\varepsilon\left({ }^{\mathcal{C}} \boldsymbol{v}_{c}+{ }^{\mathcal{C}} \boldsymbol{\omega}_{c} \times{ }^{\mathcal{C}} \boldsymbol{r}_{c}\right)$,

where ${ }^{\mathcal{C}} \boldsymbol{\omega}_{c},{ }^{\mathcal{C}} \boldsymbol{v}_{c}$, and ${ }^{\mathcal{C}} \boldsymbol{r}_{c}$ denote the chaser's angular velocity, translational velocity, and position relative to

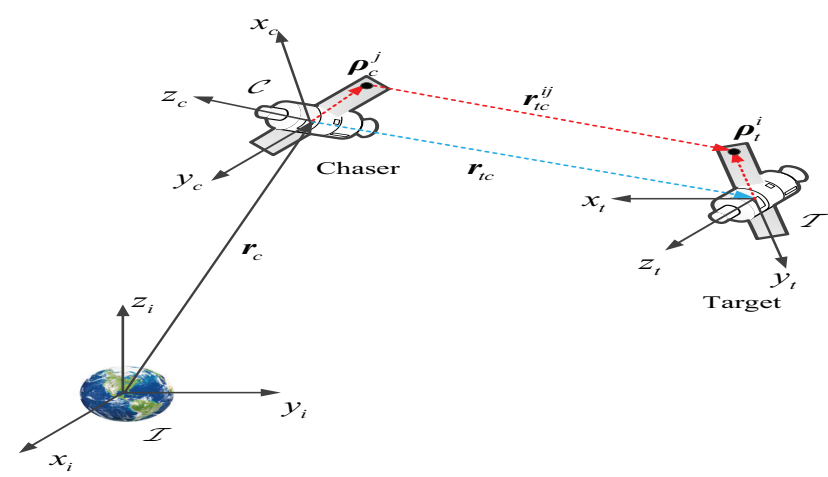

Fig. 1 The inertial and the two body-fixed reference frames.

the Earth's center of mass, respectively. All these values are expressed in the body-fixed frame $\mathcal{C}$.

The relative orientation and position of the target with respect to the chaser can be described as,

$\hat{\boldsymbol{q}}_{t c}=\hat{\boldsymbol{q}}_{c}^{*} \circ \hat{\boldsymbol{q}}_{t}=\boldsymbol{q}_{t c}+\varepsilon \frac{1}{2}{ }^{c} \boldsymbol{r}_{t c} \circ \boldsymbol{q}_{t c}$,

where $\boldsymbol{q}_{t c}$ and ${ }^{\mathcal{C}} \boldsymbol{r}_{t c}$ stand for the relative orientation and the relative position vector between the target and chaser. Similar to (1), the relative kinematic equations can be expressed by means of the dual quaternion representation,

$\dot{\hat{\boldsymbol{q}}}_{t c}=\frac{1}{2} c \hat{\boldsymbol{\omega}}_{t c} \circ \hat{\boldsymbol{q}}_{t c}$,

where ${ }^{\mathcal{C}} \hat{\boldsymbol{\omega}}_{t c}={ }^{\mathcal{C}} \boldsymbol{\omega}_{t c}+\varepsilon\left({ }^{\mathcal{C}} \boldsymbol{v}_{t c}+{ }^{\mathcal{C}} \boldsymbol{r}_{t c} \times{ }^{\mathcal{C}} \boldsymbol{\omega}_{t c}\right)$ denotes the relative velocity motor, while ${ }^{\mathcal{C}} \boldsymbol{\omega}_{t c}$ and ${ }^{\mathcal{C}} \boldsymbol{v}_{t c}$ respectively represent the relative angular velocity and translational velocity of the target in the chaser body-fixed frame $\mathcal{C}$.

The following task is to obtain the dual quaternionbased relative dynamic equations. Before proceeding, we define the dual inertia matrices of the target and chaser as $\hat{\mathbf{M}}_{a},(a=t, c)$ [37]:

$\hat{\boldsymbol{M}}_{a}=m_{a} \boldsymbol{I}_{3} \frac{d}{d \varepsilon}+\varepsilon \boldsymbol{J}_{a}, \quad \boldsymbol{J}_{a}=\left(\begin{array}{lll}J_{x x} & J_{x y} & J_{x z} \\ J_{y x} & J_{y y} & J_{y z} \\ J_{z x} & J_{z y} & J_{z z}\end{array}\right)_{a}$,

where $m_{a}$ is the mass of the rigid spacecraft, $\boldsymbol{J}_{a}$ is the matrix of the moments of inertia of the rigid spacecraft expressed in the body-fixed frame, and $\boldsymbol{I}_{3}$ is a $3 \times 3$ identity matrix. Moreover, we define the inverse of these dual inertia matrices as

$\hat{\boldsymbol{M}}_{a}^{-1}=\boldsymbol{J}_{a}^{-1} \frac{d}{d \varepsilon}+\varepsilon \frac{1}{m_{a}} \boldsymbol{I}_{3}$. 
The dual momenta of the target and chaser about their CMs, expressed in the corresponding body-fixed frame, are

$$
{ }^{\mathcal{T}} \hat{\boldsymbol{h}}_{t}=\hat{\boldsymbol{M}}_{t}{ }^{\mathcal{T}} \hat{\boldsymbol{\omega}}_{t}, \quad{ }^{\mathcal{C}} \hat{\boldsymbol{h}}_{c}=\hat{\boldsymbol{M}}_{c}{ }^{\mathcal{C}} \hat{\boldsymbol{\omega}}_{c}
$$

Differentiating (4) in their corresponding body-fixed frames $\mathcal{T}$ and $\mathcal{C}$, the absolute dynamic equations for the target and chaser are

$$
\begin{aligned}
& \mathcal{T} \dot{\hat{\boldsymbol{h}}}_{t}=\dot{\hat{\boldsymbol{M}}}_{t}{ }^{\mathcal{T}} \hat{\boldsymbol{\omega}}_{t}+\hat{\boldsymbol{M}}_{t}{ }^{\mathcal{T}} \dot{\hat{\boldsymbol{\omega}}}_{t}+{ }^{\mathcal{T}} \hat{\boldsymbol{\omega}}_{t} \times\left(\hat{\boldsymbol{M}}_{t}{ }^{\mathcal{T}} \hat{\boldsymbol{\omega}}_{t}\right)={ }^{\mathcal{T}} \hat{\boldsymbol{F}}_{t}, \\
& { }^{\mathcal{C}} \dot{\hat{\boldsymbol{h}}}_{c}=\dot{\hat{\boldsymbol{M}}}_{c}{ }^{\mathcal{C}} \hat{\boldsymbol{\omega}}_{c}+\hat{\boldsymbol{M}}_{c}{ }^{\mathcal{C}} \dot{\hat{\boldsymbol{\omega}}}_{c}+{ }^{\mathcal{C}} \hat{\boldsymbol{\omega}}_{c} \times\left(\hat{\boldsymbol{M}}_{c}{ }^{\mathcal{C}} \hat{\boldsymbol{\omega}}_{c}\right)={ }^{\mathcal{C}} \hat{\boldsymbol{F}}_{c} .
\end{aligned}
$$

where ${ }^{\mathcal{T}} \hat{\boldsymbol{F}}_{t}={ }^{\mathcal{T}} \boldsymbol{f}_{t}+\varepsilon^{\mathcal{T}} \boldsymbol{\tau}_{t}$ and ${ }^{\mathcal{C}} \hat{\boldsymbol{F}}_{c}={ }^{\mathcal{C}} \boldsymbol{f}_{c}+\varepsilon^{\mathcal{C}} \boldsymbol{\tau}_{c}$ respectively indicate the total external dual force exerting on the target and chaser. Note that these total external dual forces $\boldsymbol{F}$ contain two parts corresponding to the total external force $\boldsymbol{f}$ and torque $\boldsymbol{\tau}$.

In this study, we assume that the spacecraft mass and inertia matrix are constant, this is, $\dot{\hat{M}}_{t}=\dot{\hat{M}}_{c}=0$. Then, (5) and (6) can be rewritten as

$$
\begin{gathered}
{ }^{\mathcal{T}} \dot{\hat{\boldsymbol{\omega}}}_{t}=-\hat{\boldsymbol{M}}_{t}^{-1}\left({ }^{\mathcal{T}} \hat{\boldsymbol{\omega}}_{t} \times\left(\hat{\boldsymbol{M}}_{t}{ }^{\mathcal{T}} \hat{\boldsymbol{\omega}}_{t}\right)\right)+\hat{\boldsymbol{M}}_{t}^{-1} \mathcal{T} \hat{\boldsymbol{F}}_{t}, \\
{ }^{\mathcal{C}} \dot{\hat{\boldsymbol{\omega}}}_{c}=-\hat{\boldsymbol{M}}_{c}^{-1}\left({ }^{\mathcal{C}} \hat{\boldsymbol{\omega}}_{c} \times\left(\hat{\boldsymbol{M}}_{c}{ }^{\mathcal{C}} \hat{\boldsymbol{\omega}}_{c}\right)\right)+\hat{\boldsymbol{M}}_{c}^{-1}{ }^{\mathcal{C}} \hat{\boldsymbol{F}}_{c} .
\end{gathered}
$$

In addition, ${ }^{\mathcal{C}} \hat{\boldsymbol{\omega}}_{t c}$ can be also computed by the subtraction of the target's and chaser's absolute velocity motor, that is

${ }^{\mathcal{C}} \hat{\boldsymbol{\omega}}_{t c}=\hat{\boldsymbol{q}}_{t c} \circ{ }^{\mathcal{T}} \hat{\boldsymbol{\omega}}_{t} \circ \hat{\boldsymbol{q}}_{t c}^{*}-{ }^{\mathcal{C}} \hat{\boldsymbol{\omega}}_{c}$.

Differentiating (9) in the chaser body-fixed frame $\mathcal{C}$ and using (2), one can obtain the first-order time derivative of the relative velocity motor,

${ }^{\mathcal{C}} \dot{\hat{\boldsymbol{\omega}}}_{t c}=\hat{\boldsymbol{q}}_{t c} \circ{ }^{\mathcal{T}} \dot{\hat{\boldsymbol{\omega}}}_{t} \circ \hat{\boldsymbol{q}}_{t c}^{*}+{ }^{\mathcal{C}} \hat{\boldsymbol{\omega}}_{t c} \times\left(\hat{\boldsymbol{q}}_{t c} \circ{ }^{\mathcal{T}} \hat{\boldsymbol{\omega}}_{t} \circ \hat{\boldsymbol{q}}_{t c}^{*}\right)-{ }^{\mathcal{C}} \dot{\hat{\boldsymbol{\omega}}}_{c}$.

Substituting (7), (8), and (9) into (10), the firstorder time derivative of the relative velocity motor is further given by

$$
\begin{aligned}
\mathcal{C} \dot{\hat{\boldsymbol{\omega}}}_{t c}= & \hat{\boldsymbol{q}}_{t c} \circ\left[-\hat{\boldsymbol{M}}_{t}^{-1}\left(\hat{\boldsymbol{q}}_{t c}^{*} \circ\left({ }^{\mathcal{C}} \hat{\boldsymbol{\omega}}_{t c}+{ }^{\mathcal{C}} \hat{\boldsymbol{\omega}}_{c}\right) \circ \hat{\boldsymbol{q}}_{t c}\right)\right. \\
& \left.\times \hat{\boldsymbol{M}}_{t}\left(\hat{\boldsymbol{q}}_{t c}^{*} \circ\left({ }^{\mathcal{C}} \hat{\boldsymbol{\omega}}_{t c}+{ }^{\mathcal{C}} \hat{\boldsymbol{\omega}}_{c}\right) \circ \hat{\boldsymbol{q}}_{t c}\right)\right] \circ \hat{\boldsymbol{q}}_{t c}^{*} \\
& +{ }^{\mathcal{C}} \hat{\boldsymbol{\omega}}_{t c} \times{ }^{\mathcal{C}} \hat{\boldsymbol{\omega}}_{c}+\hat{\boldsymbol{M}}_{c}^{-1}\left({ }^{\mathcal{C}} \hat{\boldsymbol{\omega}}_{c} \times\left(\hat{\boldsymbol{M}}_{c}{ }^{\mathcal{C}} \hat{\boldsymbol{\omega}}_{c}\right)\right) \\
& +\hat{\boldsymbol{q}}_{t c} \circ \hat{\boldsymbol{M}}_{t}^{-1} \mathcal{T} \hat{\boldsymbol{F}}_{t} \circ \hat{\boldsymbol{q}}_{t c}^{*}-\hat{\boldsymbol{M}}_{c}^{-1}{ }^{\mathcal{C}} \hat{\boldsymbol{F}}_{c} .
\end{aligned}
$$

Note that (2) and (11) give us the dynamically coupled relative motion model by means of the dual quaternion representation. In particular, we can split these two formulae into their real parts, capturing the rotational motion, and their dual parts, describing the translational motion. Proceeding this way, (2) that captures the kinematic part giving the first time derivatives of the spacecraft relative position and attitude, is simply written as

${ }^{\mathcal{C}} \dot{\boldsymbol{r}}_{t c}={ }^{\mathcal{C}} \boldsymbol{v}_{t c}$,

$\dot{\boldsymbol{q}}_{t c}=\frac{1}{2} \mathcal{C} \boldsymbol{\omega}_{t c} \circ \boldsymbol{q}_{t c}$.

While (11), containing the rotational and translational dynamics, splits into

$$
\begin{aligned}
{ }^{\mathcal{C}} \dot{\boldsymbol{\omega}}_{t c}= & \boldsymbol{q}_{t c} \circ\left[-\boldsymbol{J}_{t}^{-1}\left(\boldsymbol{q}_{t c}^{*} \circ\left({ }^{\mathcal{C}} \boldsymbol{\omega}_{t c}+{ }^{\mathcal{C}} \boldsymbol{\omega}_{c}\right) \circ \boldsymbol{q}_{t c}\right)\right. \\
& \left.\times \boldsymbol{J}_{t}\left(\boldsymbol{q}_{t c}^{*} \circ\left({ }^{\mathcal{C}} \boldsymbol{\omega}_{t c}+{ }^{\mathcal{C}} \boldsymbol{\omega}_{c}\right) \circ \boldsymbol{q}_{t c}\right)\right] \circ \boldsymbol{q}_{t c}^{*} \\
& +{ }^{\mathcal{C}} \boldsymbol{\omega}_{t c} \times{ }^{\mathcal{C}} \boldsymbol{\omega}_{c}+\boldsymbol{J}_{c}^{-1}\left[{ }^{\mathcal{C}} \boldsymbol{\omega}_{c} \times\left(\boldsymbol{J}_{c}{ }^{\mathcal{C}} \boldsymbol{\omega}_{c}\right)\right] \\
& +\boldsymbol{q}_{t c} \circ\left(\boldsymbol{J}_{t}^{-1}{ }^{\mathcal{T}} \boldsymbol{\tau}_{t}\right) \circ \boldsymbol{q}_{t c}^{*}-\boldsymbol{J}_{c}^{-1 \mathcal{C}} \boldsymbol{\tau}_{c}, \\
{ }^{\mathcal{C}} \dot{\boldsymbol{v}}_{t c}= & 2{ }^{\mathcal{C}} \boldsymbol{v}_{t c} \times{ }^{\mathcal{C}} \boldsymbol{\omega}_{c}-{ }^{\mathcal{C}} \boldsymbol{\omega}_{c} \times\left({ }^{\mathcal{C}} \boldsymbol{\omega}_{c} \times{ }^{\mathcal{C}} \boldsymbol{r}_{t c}\right) \\
& +{ }^{\mathcal{C}} \boldsymbol{r}_{t c} \times \boldsymbol{J}_{c}-1\left({ }^{\mathcal{C}} \boldsymbol{\tau}_{c}-{ }^{\mathcal{C}} \boldsymbol{\omega}_{c} \times\left(\boldsymbol{J}_{c}{ }^{\mathcal{C}} \boldsymbol{\omega}_{c}\right)\right) \\
& -\frac{{ }^{\mathcal{C}} \boldsymbol{f}_{c}}{m_{c}}+\boldsymbol{q}_{t c} \circ \frac{{ }^{\mathcal{\tau}} \boldsymbol{f}_{t} \circ \boldsymbol{q}_{t c}^{*} .}{m_{t}} .
\end{aligned}
$$

It is clear that (14) is a function of the external torques ${ }^{\mathcal{T}} \boldsymbol{\tau}_{t}$ and ${ }^{\mathcal{C}} \boldsymbol{\tau}_{c}$. Since $\dot{\boldsymbol{q}}_{t c}=\frac{1}{2}{ }^{\mathcal{C}} \boldsymbol{\omega}_{t c} \circ \boldsymbol{q}_{t c}$, thus (15) explicitly depends on $\boldsymbol{q}_{t c}$ and implicitly on the external torques.

The dynamically coupled relative motion model proposed herein describes the relative motion between the CMs of two spacecraft and consists of a set of 13 dimensional state vector $\left[\boldsymbol{q}_{t c},{ }^{\mathcal{C}} \overline{\boldsymbol{r}}_{t c},{ }^{\mathcal{C}} \overline{\boldsymbol{\omega}}_{t c},{ }^{\mathcal{C}} \overline{\boldsymbol{v}}_{t c}\right]$. As for a close SFF configuration in a low Earth orbit, the total external dual force considered in this paper is decomposed as

$$
\begin{aligned}
& { }^{\mathcal{T}} \hat{\boldsymbol{F}}_{t}={ }^{\mathcal{T}} \boldsymbol{f}_{t}+\varepsilon{ }^{\mathcal{T}} \boldsymbol{\tau}_{t}=\left({ }^{\mathcal{T}} \boldsymbol{f}_{t, g}+{ }^{\mathcal{T}} \boldsymbol{f}_{t, P}\right)+\varepsilon^{\mathcal{T}} \boldsymbol{\tau}_{t, P}, \\
& { }^{\mathcal{C}} \hat{\boldsymbol{F}}_{c}={ }^{\mathcal{C}} \boldsymbol{f}_{c}+\varepsilon^{\mathcal{C}} \boldsymbol{\tau}_{c}=\left({ }^{\mathcal{C}} \boldsymbol{f}_{c, g}+{ }^{\mathcal{C}} \boldsymbol{f}_{c, P}\right)+\varepsilon{ }^{\mathcal{C}} \boldsymbol{\tau}_{c, P},
\end{aligned}
$$

where ${ }^{\mathcal{T}} \boldsymbol{f}_{t, g}$ and ${ }^{\mathcal{C}} \boldsymbol{f}_{c, g}$ respectively indicate the Earth's spherical central gravitational force acting on the target and chaser, ${ }^{\mathcal{T}} \boldsymbol{f}_{t, P}$ and ${ }^{\mathcal{C}} \boldsymbol{f}_{c, P}$ denote perturbing forces, ${ }^{\mathcal{T}} \boldsymbol{\tau}_{t, P}$ and ${ }^{\mathcal{C}} \boldsymbol{\tau}_{c, P}$ represent the total external torques.

Let ${ }^{\mathcal{A}} \boldsymbol{r}_{a}=\left[x_{a}, y_{a}, z_{a}\right]^{T},(a=t, c)$ be the position vector of the target, or of the chaser, relative to the Earth's center of mass expressed in the corresponding body-fixed frame $\mathcal{A}$ (if $a=t$, then $\mathcal{A}=\mathcal{T}$; otherwise, $\mathcal{A}=\mathcal{C})$.

The Earth's spherical central gravitational force, including its gravity gradient due to the non-infinitesimal size of the spacecraft, is given by [26],

$$
\begin{aligned}
{ }^{\mathcal{A}} \boldsymbol{f}_{a, g}= & -\frac{\mu m_{a}}{r_{a}^{3}}\left\{\boldsymbol{I}_{3}+\frac{3}{m_{a} r_{a}^{2}}\left[J_{a}+\frac{1}{2}\left(\operatorname{tr}\left(J_{a}\right)\right.\right.\right. \\
& \left.\left.\left.-5^{\mathcal{A}} \tilde{\boldsymbol{r}}_{a} J_{a}{ }^{\mathcal{A}} \tilde{\boldsymbol{r}}_{a}\right) \boldsymbol{I}_{3}\right]\right\}^{\mathcal{A}} \boldsymbol{r}_{a},
\end{aligned}
$$

where $\mu=3.986004418 \times 10^{14} \mathrm{~m}^{3} / \mathrm{s}^{2}$ is the Earth's gravitational parameter, $r_{a}$ indicates the Euclidean norm of 
the position vector ${ }^{\mathcal{A}} \boldsymbol{r}_{a}$ and ${ }^{\mathcal{A}} \tilde{\boldsymbol{r}}_{a}={ }^{\mathcal{A}} \boldsymbol{r}_{a} / r_{a}$ is its unit vector. Besides, using the chaser attitude quaternion $\boldsymbol{q}_{c}$ and the relative attitude quaternion $\boldsymbol{q}_{t c}$, the position quaternion of the target and chaser can be expressed in the $\mathcal{I}$ frame $\left({ }^{\mathcal{I}} \boldsymbol{r}_{a}=[0, x, y, z]^{T}\right)$ as

${ }^{\mathcal{I}} \boldsymbol{r}_{c}=\boldsymbol{q}_{c} \circ{ }^{\mathcal{C}} \boldsymbol{r}_{c} \circ \boldsymbol{q}_{c}^{*}, \quad{ }^{\mathcal{I}} \boldsymbol{r}_{t}=\boldsymbol{q}_{c} \circ\left(\boldsymbol{q}_{t c} \circ{ }^{\mathcal{T}} \boldsymbol{r}_{t} \circ \boldsymbol{q}_{t c}^{*}\right) \circ \boldsymbol{q}_{c}^{*}$.

The total perturbing force ${ }^{\mathcal{I}} \boldsymbol{f}_{a, P}$ we consider here induced by Earth oblateness $J_{2}$, and solar radiation pressure (SRP) are computed by means of the sum of,

$$
{ }^{\mathcal{I}} \boldsymbol{f}_{a, J_{2}}=-\frac{3}{2} J_{2} \frac{\mu R_{\oplus}^{2}}{r_{a}^{4}}\left(\begin{array}{c}
\frac{x}{r_{a}}-5 \frac{x z^{2}}{r_{a}^{3}} \\
\frac{y}{r_{a}}-5 \frac{y z^{2}}{r_{a}^{3}} \\
\frac{3 z}{r_{a}}-5 \frac{z^{3}}{r_{a}^{3}}
\end{array}\right),
$$

where $J_{2}=0.0010826267$ is the Earth's dominant zonal gravity term and $R_{\oplus}=6378.137 \mathrm{~km}$ is the Earth's equatorial radius [26], and,

${ }^{\mathcal{I}} \boldsymbol{f}_{a, s r p}=-P \frac{1 A U^{2}}{r_{s s}^{2}} \frac{A}{m} \cos (\alpha)\left[(1-\varepsilon) \boldsymbol{n}_{s}+2 \varepsilon \cos (\alpha) \boldsymbol{n}\right]$, where $P=4.56 \times 10^{-6} \mathrm{~N} / \mathrm{m}^{2}$ is the SRP force at a distance of $1 \mathrm{AU}, r_{s s}$ is the distance between the spacecraft and the Sun, $A$ is the spacecraft's cross-sectional area, $m$ is its mass, and $\varepsilon=0.3$ is the reflectivity. The unit vectors $\boldsymbol{n}_{s}$ and $\boldsymbol{n}$ indicate the Sun direction and the normal direction of the spacecraft cross sectional area $A$, respectively, thus $\cos (\alpha)=\boldsymbol{n} \cdot \boldsymbol{n}_{s}[27]$.

In addition, the total torque induced by Earth's gravity-gradient and SRP can be expressed in the bodyfixed frame $\mathcal{A}$ as ${ }^{\mathcal{A}} \boldsymbol{\tau}_{a, P}={ }^{\mathcal{A}} \boldsymbol{\tau}_{a, \nabla g}+{ }^{\mathcal{A}} \boldsymbol{\tau}_{a, s r p}$. The detailed formulae of the Earth's gravity-gradient torque ${ }^{\mathcal{A}} \boldsymbol{\tau}_{a, \nabla g}$ and SRP torque ${ }^{\mathcal{A}} \boldsymbol{\tau}_{a, s r p}$ are

${ }^{\mathcal{A}} \boldsymbol{\tau}_{a, \nabla g}=\frac{3 \mu}{r_{a}^{5}}{ }^{\mathcal{A}} \boldsymbol{r}_{a} \times \boldsymbol{J}_{a}^{\mathcal{A}} \boldsymbol{r}_{a}$

${ }^{\mathcal{A}} \boldsymbol{\tau}_{a, s r p}={ }^{\mathcal{A}} \boldsymbol{r}_{a, s r p} \times{ }^{\mathcal{A}} \boldsymbol{f}_{a, s r p}$,

where ${ }^{\mathcal{A}} \boldsymbol{r}_{a, s r p}$ indicates the position vector from the $\mathrm{CM}$ to the point where the SRP is applied.

\subsection{Six-DOF coupled relative motion model}

Consider the relative translational motion between two feature points respectively located on the target and chaser, as shown in Fig. 1. Assume $\boldsymbol{\rho}_{t}^{i}$ be an arbitrary point on the target and its position vector in the target body-fixed frame $\mathcal{T}$ is denoted as ${ }^{\mathcal{T}} \boldsymbol{\rho}_{t}^{i}=\left[\rho_{t x}^{i}, \rho_{t y}^{i}, \rho_{t z}^{i}\right]^{T}$. Analogously, let $\rho_{c}^{j}$ be an arbitrary point on the chaser, with its position vector in the chaser body-fixed frame
$\mathcal{C}$ denoted by ${ }^{\mathcal{C}} \boldsymbol{\rho}_{c}^{j}=\left[\rho_{c x}^{j}, \rho_{c y}^{j}, \rho_{c z}^{j}\right]^{T}$. As a particular case, the position vectors of the CMs of the target and chaser can be represented by ${ }^{\mathcal{T}} \boldsymbol{\rho}_{t}^{0}=[0,0,0]^{T},{ }^{\mathcal{C}} \boldsymbol{\rho}_{c}^{0}=$ $[0,0,0]^{T}$. Clearly, one can obtain the relative position vector between two arbitrary points $\boldsymbol{\rho}_{t}^{i}$ and $\boldsymbol{\rho}_{c}^{j}$ as

$\boldsymbol{r}_{t c}^{i j}=\boldsymbol{r}_{t c}+\boldsymbol{\rho}_{t}^{i}-\boldsymbol{\rho}_{c}^{j}$,

where $\boldsymbol{r}_{t c}$ indicates the relative position between two CMs. Moreover, since the target and chaser are regarded as rigid-body spacecraft, thus

$\left.\frac{d \boldsymbol{\rho}_{t}^{i}}{d t}\right|_{\mathcal{T}}=0,\left.\quad \frac{d \boldsymbol{\rho}_{c}^{j}}{d t}\right|_{\mathcal{C}}=0$,

where the notations $\left.\frac{d}{d t}\right|_{\mathcal{T}}$ and $\left.\frac{d}{d t}\right|_{\mathcal{C}}$ stand for the time derivatives computed in the frames $\mathcal{T}$ and $\mathcal{C}$.

Then, using (12) and (17), the first and second order time derivatives of $\boldsymbol{r}_{t c}^{i j}$ in the frame $\mathcal{C}$ are given as

$$
\begin{aligned}
{ }^{\mathcal{C}} \boldsymbol{v}_{t c}^{i j}= & { }^{\mathcal{C}} \boldsymbol{v}_{t c}+{ }^{\mathcal{C}} \boldsymbol{\omega}_{t c} \times\left(\boldsymbol{q}_{t c} \circ{ }^{\mathcal{T}} \boldsymbol{\rho}_{t}^{i} \circ \boldsymbol{q}_{t c}^{*}\right), \\
{ }^{\mathcal{C}} \dot{\boldsymbol{v}}_{t c}^{i j}= & { }^{\mathcal{C}} \dot{\boldsymbol{v}}_{t c}+{ }^{\mathcal{C}} \dot{\boldsymbol{\omega}}_{t c} \times\left(\boldsymbol{q}_{t c} \circ{ }^{\mathcal{T}} \boldsymbol{\rho}_{t}^{i} \circ \boldsymbol{q}_{t c}^{*}\right) \\
& +{ }^{\mathcal{C}} \boldsymbol{\omega}_{t c} \times\left({ }^{\mathcal{C}} \boldsymbol{\omega}_{t c} \times\left(\boldsymbol{q}_{t c} \circ{ }^{\mathcal{T}} \boldsymbol{\rho}_{t}^{i} \circ \boldsymbol{q}_{t c}^{*}\right)\right) .
\end{aligned}
$$

Finally, substituting (15), (16), and (18) into (19), the relative translational motion between two arbitrary points on the target and chaser is captured by

$$
\begin{aligned}
{ }^{\mathcal{C}} \dot{\boldsymbol{v}}_{t c}^{i j}= & \left({ }^{\mathcal{C}} \boldsymbol{r}_{t c}^{i j}-\boldsymbol{q}_{t c} \circ{ }^{\mathcal{T}} \boldsymbol{\rho}_{t}^{i} \circ \boldsymbol{q}_{t c}^{*}+{ }^{\mathcal{C}} \boldsymbol{\rho}_{c}^{j}\right) \times \boldsymbol{J}_{c}^{-1}\left({ }^{\mathcal{C}} \boldsymbol{\tau}_{c}\right. \\
& \left.-{ }^{\mathcal{C}} \boldsymbol{\omega}_{c} \times \boldsymbol{J}_{c}{ }^{\mathcal{C}} \boldsymbol{\omega}_{c}\right)+{ }^{\mathcal{C}} \dot{\boldsymbol{\omega}}_{t c} \times\left(\boldsymbol{q}_{t c} \circ{ }^{\mathcal{T}} \boldsymbol{\rho}_{t}^{i} \circ \boldsymbol{q}_{t c}^{*}\right) \\
& +2\left[{ }^{\mathcal{C}} \boldsymbol{v}_{t c}^{i j}-{ }^{\mathcal{C}} \boldsymbol{\omega}_{t c} \times\left(\boldsymbol{q}_{t c} \circ{ }^{\mathcal{T}} \boldsymbol{\rho}_{t}^{i} \circ \boldsymbol{q}_{t c}^{*}\right)\right] \times{ }^{\mathcal{C}} \boldsymbol{\omega}_{c} \\
& -{ }^{\mathcal{C}} \boldsymbol{\omega}_{c} \times\left[{ }^{\mathcal{C}} \boldsymbol{\omega}_{c} \times\left({ }^{\mathcal{C}} \boldsymbol{r}_{t c}^{i j}-\boldsymbol{q}_{t c} \circ{ }^{\mathcal{T}} \boldsymbol{\rho}_{t}^{i} \circ \boldsymbol{q}_{t c}^{*}+\right.\right. \\
& \left.\left.{ }^{\mathcal{C}} \boldsymbol{\rho}_{c}^{j}\right)\right]+{ }^{\mathcal{C}} \boldsymbol{\omega}_{t c} \times\left({ }^{\mathcal{C}} \boldsymbol{\omega}_{t c} \times\left(\boldsymbol{q}_{t c} \circ{ }^{\mathcal{T}} \boldsymbol{\rho}_{t}^{i} \circ \boldsymbol{q}_{t c}^{*}\right)\right) \\
& -\frac{{ }^{\mathcal{C}} \boldsymbol{f}_{c}}{m_{c}}+\boldsymbol{q}_{t c} \circ \frac{{ }^{\mathcal{T}} \boldsymbol{f}_{t}}{m_{t}} \circ \boldsymbol{q}_{t c}^{*} .
\end{aligned}
$$

It is worth to mention that, in order to compute the external dual force acting on the target, one should first compute its position vector expressed in frame $\mathcal{T}$. Then, using (16), one obtains

$$
{ }^{\mathcal{T}} \boldsymbol{r}_{t}=\boldsymbol{q}_{t c}^{*} \circ\left({ }^{\mathcal{C}} \boldsymbol{r}_{c}+{ }^{\mathcal{C}} \boldsymbol{r}_{t c}^{i j}+{ }^{\mathcal{C}} \boldsymbol{\rho}_{c}^{j}\right) \circ \boldsymbol{q}_{t c}-{ }^{\mathcal{T}} \boldsymbol{\rho}_{t}^{i} .
$$

As a result of the preceding discussions, we have obtained a six-DOF relative motion model of two arbitrary feature points located on the chaser and target, which consists of equations (13), (14), (18), (20) with a set of 13 dimensional state vector $\left[\boldsymbol{q}_{t c},{ }^{\mathcal{C}} \overline{\boldsymbol{r}}_{t c}^{i j},{ }^{\mathcal{C}} \overline{\boldsymbol{\omega}}_{t c},{ }^{\mathcal{C}} \overline{\boldsymbol{v}}_{t c}^{i j}\right]$. It is clear that (18) and (20) do not use the regular angular velocity and acceleration of the local-verticallocal-horizontal reference frame fixed to the chaser's $\mathrm{CM}$ and generalizes results based on Keplerian orbits. Moreover, it allows the analysis of both kinematic and 
dynamic coupling effects on the relative translation under the consideration of arbitrary external perturbations. But of course, we need to include the chaser's equations of motion (1) and (8) for the propagation of the perturbed chaser orbit. As an illustrative example, we consider $J_{2}$ in the following simulations to validate its effectiveness in the perturbed reference orbit case.

\section{Simulations}

Assuming two close spacecraft orbiting the Earth, the purpose of this section is to validate the feasibility of the proposed model in order to analize both the kinematic and dynamic coupling effects on the relative translational motion, as well as the impact of orbital perturbations. We study the kinematic coupling effect induced by the displacements of two non-CM feature points in both the Keplerian and perturbed orbit cases.

The dynamic coupling effect induced by forces or torques depends on the attitude motion of the spacecraft. For brevity, here we only consider the SRP acceleration and its associated torque, other forces and torques, also responsible of the dynamic coupling effect, could be managed in a similar way. We note that the dynamic coupling effect induced by the $J_{2}$ perturbation is tiny and negligible, therefore, the real impact of the $J_{2}$ perturbation on the relative translational motion stems from the differential dynamics, and in what follows, it will referred as the $J_{2}$ perturbation effect.

We carry out four numerical simulations. The first one considers a SFF flying in Keplerian orbits, and it is devoted to analyze the kinematic coupling effect on the relative translational motion due to the displacements of the feature points. The second one analyzes the kinematic coupling effect in the $J_{2}$ perturbed orbital case, which has been rarely investigated. The third numerical simulation compares the kinematic coupling effect with the $J_{2}$ perturbation effect induced by the differential dynamics. Finally, the last simulation considers the dynamic coupling effect on the relative translational motion induced by the SRP, whose magnitude depends on the spacecraft attitude, and we also include a comparative with the $J_{2}$ perturbation effect.

\subsection{Kinematic coupling effect in the Keplerian case}

In this case study we assume that the chaser operates on an ideal Keplerian elliptic orbit with semi-major axis $a_{c}=7170 \mathrm{~km}$, eccentricity $e_{c}=0.05$, inclination $i_{c}=15 \mathrm{deg}$, right ascension of the ascending node $\Omega_{c}=0 \mathrm{deg}$, argument of perigee $\omega_{c}=340 \mathrm{deg}$, and true anomaly $\theta_{c}=20 \mathrm{deg}$. The chaser's initial attitude quaternion is $\boldsymbol{q}_{c}(0)=[1,0,0,0]^{T}$. Then, converting the selected orbital elements into the chaser position and velocity vectors, we get: ${ }^{\mathcal{C}} \boldsymbol{r}_{c}=[6831117.5,0,0]^{T} \mathrm{~m}$, ${ }^{\mathcal{C}} \boldsymbol{v}_{c}=[127.67,446.17,2022.97]^{T} \mathrm{~m} / \mathrm{s}$.

To achieve a close SFF orbiting the Earth, we derive the initial relative position and velocity between the CMs of the target and chaser by means of the energy matching condition [38], i.e. ${ }^{\mathcal{C}} \boldsymbol{r}_{t c}=[40,23.11,68.31]^{T} \mathrm{~m}$, ${ }^{\mathcal{C}} \boldsymbol{v}_{t c}=[0,-0.0857,-0.0230]^{T} \mathrm{~m} / \mathrm{s}$. The initial relative attitude quaternion is chosen as $\boldsymbol{q}_{t c}=\left[\frac{\sqrt{2}}{2}, \frac{\sqrt{2}}{2}, 0,0\right]^{T}$. Moreover, the inertia tensors for the target and chaser are assumed to be: $\boldsymbol{J}_{t}=\boldsymbol{J}_{c}=\operatorname{diag}[500 ; 600 ; 700] \mathrm{kg} \cdot \mathrm{m}^{2}$.

With the purpose of investigating the kinematic coupling effect on the spacecraft relative translational motion, we consider four different scenarios choosing different initial values of the chaser angular, and relative angular, velocities. The two selected initial angular velocities of the chaser are $:{ }^{\mathcal{C}} \boldsymbol{\omega}_{c}=[0,0,0.0010]^{T}$ $\mathrm{rad} / \mathrm{s}$, (small initial chaser angular velocity), and ${ }^{\mathcal{C}} \boldsymbol{\omega}_{c}=$ $[0,0.0104,0.0104]^{T} \mathrm{rad} / \mathrm{s}$, (large initial chaser angular velocity). The two selected initial relative angular velocities are: ${ }^{\mathcal{C}} \boldsymbol{\omega}_{t c}=[0,0.0014,-0.0008]^{T} \mathrm{rad} / \mathrm{s}$, (small initial relative angular velocity), and ${ }^{\mathcal{C}} \boldsymbol{\omega}_{t c}=$ $[0,0.0140,-0.0081]^{T} \mathrm{rad} / \mathrm{s}$, (large initial relative angular velocity). In what follows, we denote the relative position between the CMs of the target and chaser by $\boldsymbol{r}_{t c}^{00}$ instead of $\boldsymbol{r}_{t c}$.

In this case study no external perturbations are considered. We employ the proposed nonlinear coupled relative motion model to simulate the translational motion between two feature points on the target: ${ }^{\mathcal{T}} \boldsymbol{\rho}_{t}^{i}, i=0,1$, and two feature points on the chaser: ${ }^{\mathcal{C}} \boldsymbol{\rho}_{c}^{j}, j=0,1$. The corresponding position vectors of these four feature points are ${ }^{\mathcal{T}} \boldsymbol{\rho}_{t}^{0}=[0,0,0],{ }^{\mathcal{T}} \boldsymbol{\rho}_{t}^{1}=[-2,2,-2] \mathrm{m}$ and ${ }^{\mathcal{C}} \boldsymbol{\rho}_{c}^{0}=[0,0,0],{ }^{\mathcal{C}} \boldsymbol{\rho}_{c}^{1}=[-2,2,2] \mathrm{m}$. We compute the initial relative position and velocity vectors between the feature points using (16) and (18), respectively.

Figure 2 displays four SFF relative motion trajectories, ${ }^{\mathcal{I}} \boldsymbol{r}_{t c}^{00},{ }^{\mathcal{I}} \boldsymbol{r}_{t c}^{10},{ }^{\mathcal{I}} \boldsymbol{r}_{t c}^{01}$, and ${ }^{\mathcal{I}} \boldsymbol{r}_{t c}^{11}$, in the inertial frame $\mathcal{I}$ for one orbital period, and for sake of brevity, we are going to omit the pre-superscript $\mathcal{I}$ in the following discussions. The differences among the four plots are the initial conditions for chaser angular, and relative angular, velocities. The results show that the relative motion between two non-CM feature points on the target and chaser does not coincide with the one between their CMs. Furthermore, it also reveals that remarkable harmonic oscillations are generated due to the kinematic coupling effect. Figure 3 displays the history of relative position deviations, i.e. $\delta \boldsymbol{r}_{t c}^{10}=\boldsymbol{r}_{t c}^{10}-\boldsymbol{r}_{t c}^{00}$, $\delta \boldsymbol{r}_{t c}^{01}=\boldsymbol{r}_{t c}^{01}-\boldsymbol{r}_{t c}^{00}, \delta \boldsymbol{r}_{t c}^{11}=\boldsymbol{r}_{t c}^{11}-\boldsymbol{r}_{t c}^{00}$. The oscillation frequencies of the relative position deviations in Figs. 3(b), 

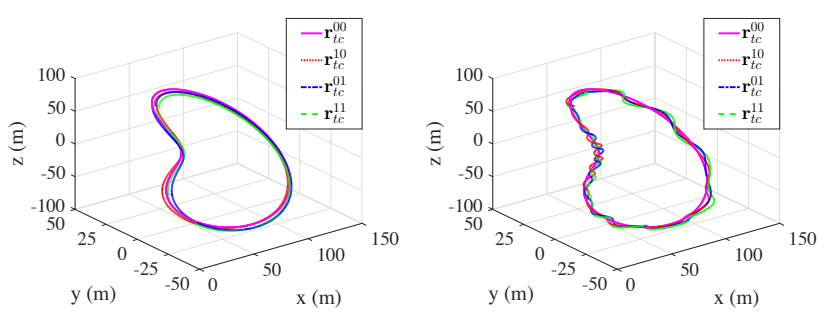

(a) small ${ }^{\mathcal{C}} \boldsymbol{\omega}_{c}$, small ${ }^{\mathcal{C}} \boldsymbol{\omega}_{t c}$

(b) large ${ }^{\mathcal{C}} \boldsymbol{\omega}_{c}$, small ${ }^{\mathcal{C}} \boldsymbol{\omega}_{t c}$
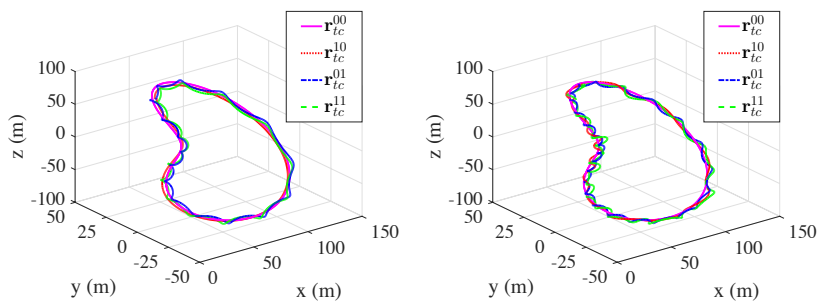

(c) small ${ }^{\mathcal{C}} \boldsymbol{\omega}_{c}$, large ${ }^{\mathcal{C}} \boldsymbol{\omega}_{t c}$

(d) large ${ }^{\mathcal{C}} \boldsymbol{\omega}_{c}$, large ${ }^{\mathcal{C}} \boldsymbol{\omega}_{t c}$

Fig. 2 Relative motion trajectories in the Keplerian case.

3(c), and 3(d) are higher than that in Fig. 3(a) due to the faster chaser angular, or relative angular, velocities.

4.2 Kinematic coupling effect in the $J_{2}$ perturbed case

In this case study we show the effectiveness of the proposed coupled relative motion model to analize the kinematic coupling effect on the relative translation, when a SFF operates in perturbed orbits. Without loss of generality, we consider the chaser and target in nonKeplerian $J_{2}$ perturbed orbits. The remaining simulation conditions and parameters coincide with the ones of the previous Keplerian case.

Figure 4 exhibits the evolutions of four SFF trajectories: ${ }_{2} \boldsymbol{r}_{t c}^{00},{ }_{J_{2}} \boldsymbol{r}_{t c}^{10}, J_{2} \boldsymbol{r}_{t c}^{01}$, and ${ }_{J_{2}} \boldsymbol{r}_{t c}^{11}$ under different initial chaser angular, and relative angular, velocities for one orbital period. Figure 5 displays the deviations in the relative positions for the non-CM feature points, defined as ${ }_{J_{2}} \delta \boldsymbol{r}_{t c}^{10}={ }_{J_{2}} \boldsymbol{r}_{t c}^{10}-J_{2} \boldsymbol{r}_{t c}^{00},{ }_{J_{2}} \delta \boldsymbol{r}_{t c}^{01}={ }_{J_{2}} \boldsymbol{r}_{t c}^{01}-{ }_{J_{2}} \boldsymbol{r}_{t c}^{00}$, $J_{2} \delta \boldsymbol{r}_{t c}^{11}={ }_{J_{2}} \boldsymbol{r}_{t c}^{11}-J_{2} \boldsymbol{r}_{t c}^{00}$. Comparisons between Fig. 2 and Fig. 4, Fig. 3 and Fig. 5 show that the kinematic cou-
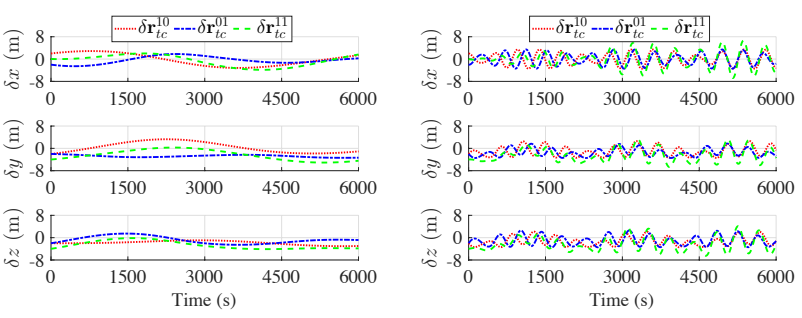

(a) small ${ }^{\mathcal{C}} \boldsymbol{\omega}_{c}$, small ${ }^{\mathcal{C}} \boldsymbol{\omega}_{t c}$

(b) large ${ }^{\mathcal{C}} \boldsymbol{\omega}_{c}$, small ${ }^{\mathcal{C}} \boldsymbol{\omega}_{t c}$
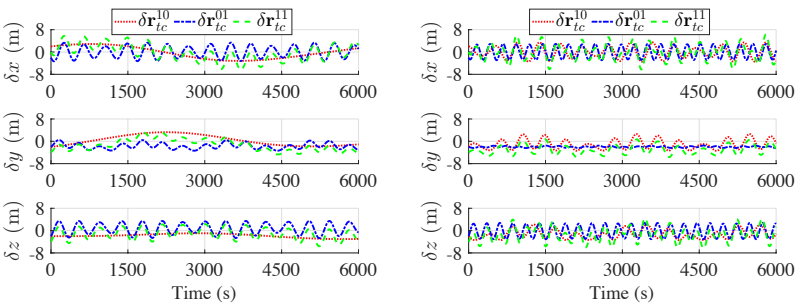

(c) small ${ }^{\mathcal{C}} \boldsymbol{\omega}_{c}$, large ${ }^{\mathcal{C}} \boldsymbol{\omega}_{t c}$

(d) large ${ }^{\mathcal{C}} \boldsymbol{\omega}_{c}$, large ${ }^{\mathcal{C}} \boldsymbol{\omega}_{t c}$

Fig. 3 Relative position deviations between $\boldsymbol{r}_{t c}^{10}$ and $\boldsymbol{r}_{t c}^{00}, \boldsymbol{r}_{t c}^{01}$ and $\boldsymbol{r}_{t c}^{00}, \boldsymbol{r}_{t c}^{11}$ and $\boldsymbol{r}_{t c}^{00}$ in the Keplerian case.

pling effect on the spacecraft relative translation motion in the $J_{2}$ perturbed case is similar to the one of the Keplerian case. Remarkable harmonic oscillations still exist in Figs. 5(b), 5(c) and 5(d), and with a higher oscillation frequencies than those in Fig. 5(a). It is worth to mention that these numerical simulations show that the proposed coupled relative motion model is effective in the perturbed environment. Essentially, the proposed model generalizes the work in $[5,6]$, that is, it enables to analyze the kinematic coupling effect in a general manner.

4.3 Comparison between the kinematic coupling and the $J_{2}$ perturbation effects

The aim of this case study is to compare the magnitudes of the kinematic coupling effect and the $J_{2}$ perturbation effect on the relative translation motion between two arbitrary feature points. Again, four relative translational trajectories $\left(\boldsymbol{r}_{t c}^{00}, J_{2} \boldsymbol{r}_{t c}^{00}, \boldsymbol{r}_{t c}^{11}\right.$, and $\left.{ }_{J_{2}} \boldsymbol{r}_{t c}^{11}\right)$ 

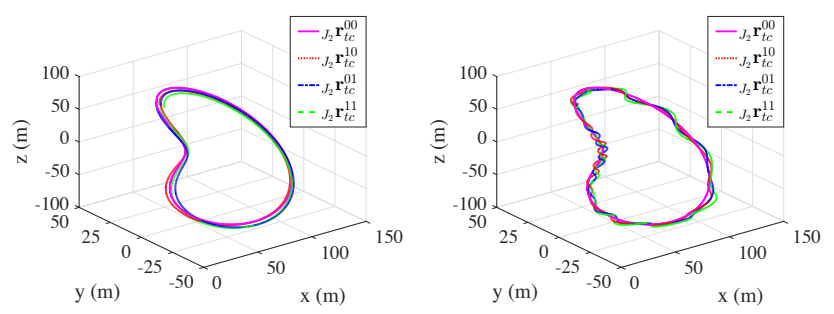

(a) small ${ }^{\mathcal{C}} \boldsymbol{\omega}_{c}$, small ${ }^{\mathcal{C}} \boldsymbol{\omega}_{t c}$

(b) large ${ }^{\mathcal{C}} \boldsymbol{\omega}_{c}$, small ${ }^{\mathcal{C}} \boldsymbol{\omega}_{t c}$
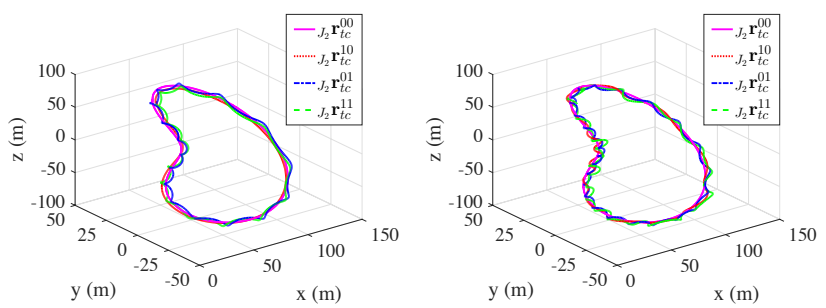

(c) small ${ }^{\mathcal{C}} \boldsymbol{\omega}_{c}$, large ${ }^{\mathcal{C}} \boldsymbol{\omega}_{t c}$

(d) large ${ }^{\mathcal{C}} \boldsymbol{\omega}_{c}$, large ${ }^{\mathcal{C}} \boldsymbol{\omega}_{t c}$

Fig. 4 Relative motion trajectories in the $J_{2}$ perturbed case.

are propagated in two main scenarios, including, small and large, initial chaser angular, and relative angular, velocities. Note that $\boldsymbol{r}_{t c}^{00}$ and ${ }_{J_{2}} \boldsymbol{r}_{t c}^{00}$ indicate the propagation of the relative position between the CMs of the target and chaser, in the Keplerian orbit case and in the $J_{2}$ perturbed orbit case, respectively. Analogously, $\boldsymbol{r}_{t c}^{11}$ and ${ }_{J_{2}} \boldsymbol{r}_{t c}^{11}$ indicate the propagation of relative motion between $\boldsymbol{\rho}_{t}^{1}$ and $\boldsymbol{\rho}_{c}^{1}$ in their respective cases. Note that the difference between $\boldsymbol{r}_{t c}^{11}$ and $\boldsymbol{r}_{t c}^{00}$ indicates the kinematic coupling effect, i.e., $\Delta \boldsymbol{r}_{t c}^{11}=\boldsymbol{r}_{t c}^{11}-\boldsymbol{r}_{t c}^{00}$. The difference between ${ }_{J_{2}} \boldsymbol{r}_{t c}^{00}$ and $\boldsymbol{r}_{t c}^{00}$ denotes the $J_{2}$ perturbation effect, $\Delta_{J_{2}} \boldsymbol{r}_{t c}^{00}={ }_{J_{2}} \boldsymbol{r}_{t c}^{00}-\boldsymbol{r}_{t c}^{00}$. Besides, the deviation $\Delta_{J_{2}} \boldsymbol{r}_{t c}^{11}={ }_{J_{2}} \boldsymbol{r}_{t c}^{11}-\boldsymbol{r}_{t c}^{00}$ represents the total behavior of both the kinematic coupling and $J_{2}$ perturbation effects.

In this case study four relative translational trajectories $\boldsymbol{r}_{t c}^{00},{ }_{J_{2}} \boldsymbol{r}_{t c}^{00}, \boldsymbol{r}_{t c}^{11}$, and ${ }_{J_{2}} \boldsymbol{r}_{t c}^{11}$ are propagated for 10 hours. For the sake of brevity, Figs. 6(a) and 7(a) merely show the relative motion trajectories over one orbital period. Figures 6(b) and 7(b) show the time history of $\Delta \boldsymbol{r}_{t c}^{11}$, indicating the kinematic coupling effect on the
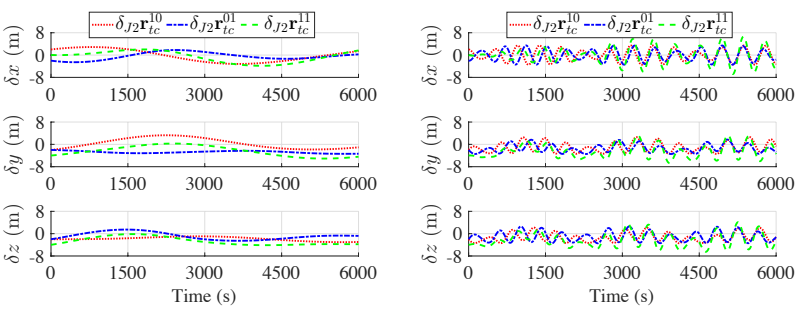

(a) small ${ }^{\mathcal{C}} \boldsymbol{\omega}_{c}$, small ${ }^{\mathcal{C}} \boldsymbol{\omega}_{t c}$

(b) large ${ }^{\mathcal{C}} \boldsymbol{\omega}_{c}$, small ${ }^{\mathcal{C}} \boldsymbol{\omega}_{t c}$
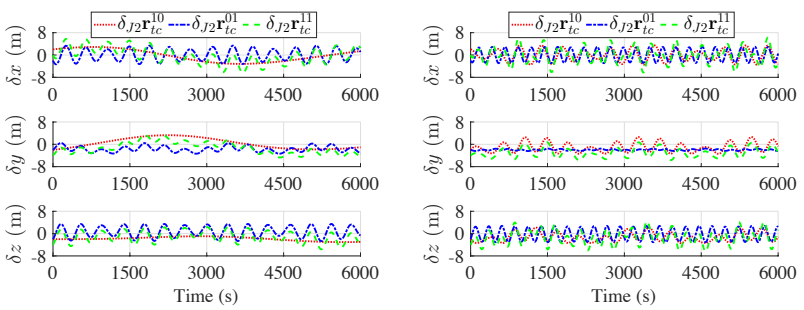

(c) small ${ }^{\mathcal{C}} \boldsymbol{\omega}_{c}$, large ${ }^{\mathcal{C}} \boldsymbol{\omega}_{t c}$

(d) large ${ }^{\mathcal{C}} \boldsymbol{\omega}_{c}$, large ${ }^{\mathcal{C}} \boldsymbol{\omega}_{t c}$

Fig. 5 Relative position deviations between ${ }_{J_{2}} \boldsymbol{r}_{t c}^{10}$ and ${ }_{J_{2}} \boldsymbol{r}_{t c}^{00}$, ${ }_{J_{2}} \boldsymbol{r}_{t c}^{01}$ and ${ }_{J_{2}} \boldsymbol{r}_{t c}^{00}, J_{2} \boldsymbol{r}_{t c}^{11}$ and ${ }_{J_{2}} \boldsymbol{r}_{t c}^{00}$ in the J2 perturbed case.

relative translation in the Keplerian orbital case. It is apparent that the kinematic coupling effect is bounded. Similar to the conclusions obtained in the previous two case studies, a comparison between Figs. 6(b) and 7(b) reveals that a large initial chaser angular velocity and a large initial relative angular velocity lead to remarkable harmonic oscillations. Figures 6(c) and 7(c) describe the time history of $\Delta_{J_{2}} \boldsymbol{r}_{t c}^{00}$, which merely exhibit the $J_{2}$ perturbation effect on the relative translation between the CMs of two spacecraft. The kinematic coupling effect is eliminated through fixing the feature points on the CMs of the target and chaser. It is worth to mention that Figs. 6(c) and 7(c) are the same, showing that the $J_{2}$ perturbation effect is independent on the rotations of the target and chaser. Besides, the $J_{2}$ perturbation effect increases gradually with time. The comparisons between Figs. 6(b) and 6(c), Figs. 7(b) and 7(c) show that the kinematic coupling effect is more dominant in the initial propagation phase, while the $J_{2}$ perturbation effect is more significant in long operation times (typ- 

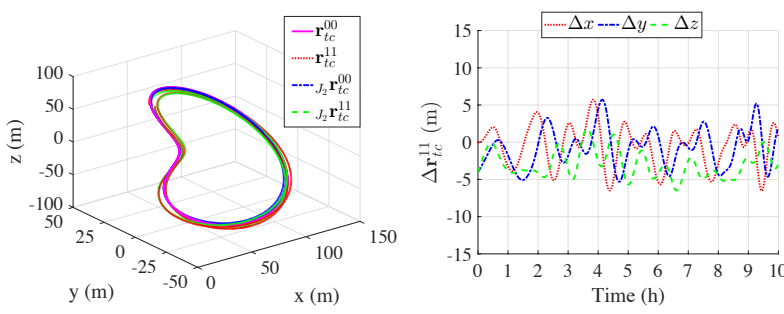

(a) relative trajectories
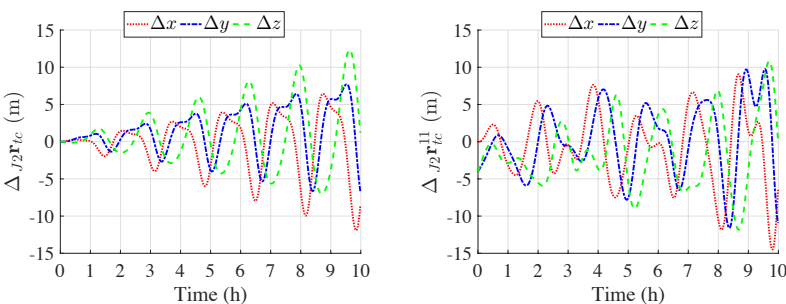

(c) $\Delta_{J_{2}} \boldsymbol{r}_{t c}^{00}={ }_{J_{2}} \boldsymbol{r}_{t c}^{00}-\boldsymbol{r}_{t c}^{00}$

(d) $\Delta_{J_{2}} \boldsymbol{r}_{t c}^{11}=J_{2} \boldsymbol{r}_{t c}^{11}-\boldsymbol{r}_{t c}^{00}$

Fig. 6 Analyses of both kinematic coupling and $J_{2}$ perturbation effects on the relative translational motion for small initial chaser angular velocity ${ }^{\mathcal{C}} \boldsymbol{\omega}_{c}$ and relative angular velocity ${ }^{\mathcal{C}} \omega_{t c}$.

ical, for instance, during a long-time tight SFF mission). Therefore, the $J_{2}$ perturbation effect generally can not be omitted as compared to the kinematic coupling effect. Finally, Figs. 6(d) and 7(d) show the total behavior of both the kinematic coupling and $J_{2}$ perturbation effects on the relative translation. Essentially, it is the superposition of both relative position deviations respectively induced by the kinematic coupling feature and the $J_{2}$ perturbation.

\subsection{Analysis of the dynamic coupling effect}

In this last case study, we address the dynamic coupling effect on the relative translational motion using the proposed coupled relative motion model and we also discuss the results comparing with the $J_{2}$ perturbation effect. As an illustrative example, we only consider the dynamic coupling effect induced by the SRP with the
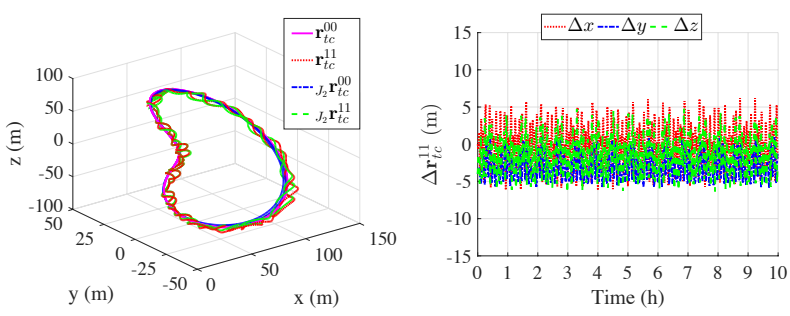

(a) relative trajectories

(b) $\Delta \boldsymbol{r}_{t c}^{11}=\boldsymbol{r}_{t c}^{11}-\boldsymbol{r}_{t c}^{00}$
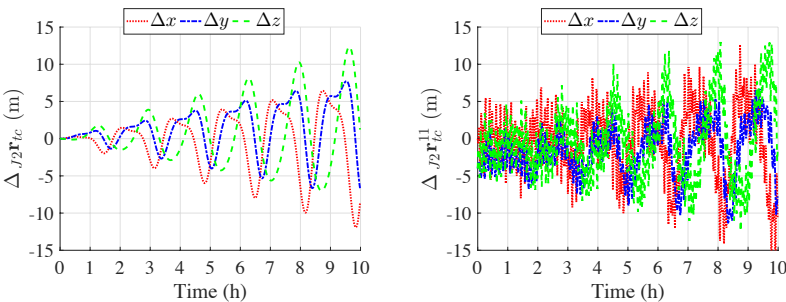

(c) $\Delta_{J_{2}} \boldsymbol{r}_{t c}^{00}={ }_{J_{2}} \boldsymbol{r}_{t c}^{00}-\boldsymbol{r}_{t c}^{00}$

(d) $\Delta_{J_{2}} \boldsymbol{r}_{t c}^{11}={ }_{J_{2}} \boldsymbol{r}_{t c}^{11}-\boldsymbol{r}_{t c}^{00}$

Fig. 7 Analyses of both kinematic coupling and $J_{2}$ perturbation effects on the relative translational motion for large initial chaser angular velocity ${ }^{\mathcal{C}} \boldsymbol{\omega}_{c}$ and relative angular velocity ${ }^{\mathcal{C}} \boldsymbol{\omega}_{t c}$.

area- to-mass ratios of the chaser and target taken as $0.01 \mathrm{~m}^{2} / \mathrm{kg}$. Without loss of generality, we take into account two representative scenarios by means of assuming whether the spacecraft is rotating in the inertial frame or not. In the first scenario, the attitude of both the chaser and target are fixed in the inertial frame, and the relative position vector between their $\mathrm{CMs}$ is denoted as ${ }_{f} \boldsymbol{r}_{t c}^{00}$. In the second scenario, the initial chaser angular, and relative angular velocity vectors are respectively set to ${ }^{\mathcal{C}} \boldsymbol{\omega}_{c}=[0,0.0104,0.0104]^{T} \mathrm{rad} / \mathrm{s}$, and ${ }^{\mathcal{C}} \boldsymbol{\omega}_{t c}=[0,0.0140,-0.0081]^{T} \mathrm{rad} / \mathrm{s}$. In this scenario, we denote the relative position vector between the CMs of the chaser and target by ${ }_{c} \boldsymbol{r}_{t c}^{00}$. Apart from this, all the other simulation conditions and parameters coincide with the ones adopted in the previous Keplerian case.

In Fig. 8 we exhibit the evolutions of two SFF relative trajectories ${ }_{c} \boldsymbol{r}_{t c}^{00}$ and ${ }_{f} \boldsymbol{r}_{t c}^{00}$, as well as their deviation $\Delta_{s r p} \boldsymbol{r}_{t c}^{00}={ }_{c} \boldsymbol{r}_{t c}^{00}-{ }_{f} \boldsymbol{r}_{t c}^{00}$. The history of this deviation 

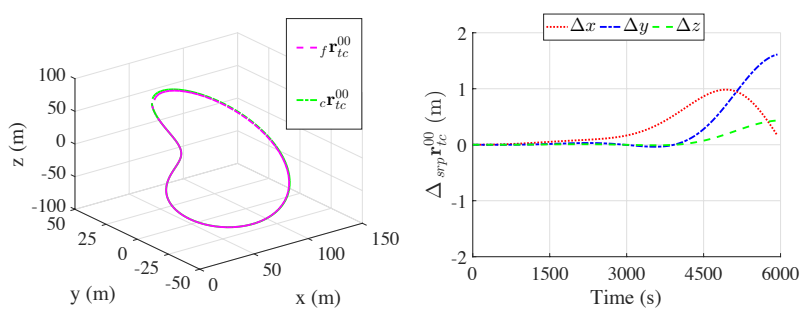

(a) relative trajectories

(b) $\Delta_{s r p} \boldsymbol{r}_{t c}^{00}={ }_{c} \boldsymbol{r}_{t c}^{00}-{ }_{f} \boldsymbol{r}_{t c}^{00}$

Fig. 8 Relative trajectories and position deviation between ${ }_{c} \boldsymbol{r}_{t c}^{00}$ and ${ }_{f} \boldsymbol{r}_{t c}^{00}$.

vector indicates the dynamic coupling effect on the relative translational motion. Apparently, it is unbounded and increases gradually with time. Again, when compared with the bounded kinematic coupling effect, the dynamic coupling effect becomes significant in a longtime tight SFF mission.

The comparison between the dynamic coupling effect and $J_{2}$ perturbation effect has been also investigated. We display the propagations of the four relative translational trajectories, ${ }_{f} \boldsymbol{r}_{t c}^{00},{ }_{c} \boldsymbol{r}_{t c}^{00},{ }_{f, J_{2}} \boldsymbol{r}_{t c}^{00}$ and ${ }_{c, J_{2}} \boldsymbol{r}_{t c}^{00}$ in Fig. 9, where the symbols ${ }_{f} \boldsymbol{r}_{t c}^{00}$ and ${ }_{c} \boldsymbol{r}_{t c}^{00}$ denote the relative trajectories between the CMs of the target and chaser with fixed, and time-varying, attitudes. Analogously, ${ }_{f, J_{2}} \boldsymbol{r}_{t c}^{00}$ and ${ }_{c, J_{2}} \boldsymbol{r}_{t c}^{00}$ indicate the relative trajectories subjected to the $J_{2}$ perturbation. Note that the differences $\Delta_{s r p} \boldsymbol{r}_{t c}^{00}={ }_{c} \boldsymbol{r}_{t c}^{00}-{ }_{f} \boldsymbol{r}_{t c}^{00}, \Delta_{J_{2}} \boldsymbol{r}_{t c}^{00}={ }_{f, J_{2}} \boldsymbol{r}_{t c}^{00}-{ }_{f} \boldsymbol{r}_{t c}^{00}$, and $\Delta_{s r p, J_{2}} \boldsymbol{r}_{t c}^{00}={ }_{c, J_{2}} \boldsymbol{r}_{t c}^{00}-{ }_{f} \boldsymbol{r}_{t c}^{00}$ show the dynamic coupling effect induced by the SRP, the $J_{2}$ perturbation effect, and the total behavior of both the dynamic coupling effect and the $J_{2}$ perturbation. We show the histories of $\Delta_{s r p} \boldsymbol{r}_{t c}^{00}, \Delta_{J_{2}} \boldsymbol{r}_{t c}^{00}$ and $\Delta_{s r p, J_{2}} \boldsymbol{r}_{t c}^{00}$ in Figs. 9(b)9(d). Fig. 9(b) shows the dynamic coupling effect on the relative translation. In contrast to the bounded kinematic coupling, the dynamic coupling gradually increases with time. Fig. 9(c) displays the time history of $\Delta_{J_{2}} \boldsymbol{r}_{t c}^{00}$, exhibiting the accumulative behavior of the $J_{2}$ perturbation effect on the relative translational motion. The total superposition of both the dynamic coupling effect induced by the SRP and the $J_{2}$ perturbation effect is illustrated in Fig. 9(d), which exhibits characteristics that increase over time.
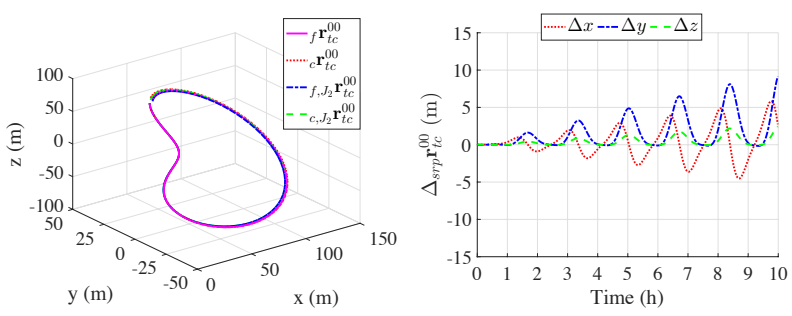

(a) relative trajectories

(b) $\Delta_{s r p} \boldsymbol{r}_{t c}^{00}={ }_{c} \boldsymbol{r}_{t c}^{00}-{ }_{f} \boldsymbol{r}_{t c}^{00}$
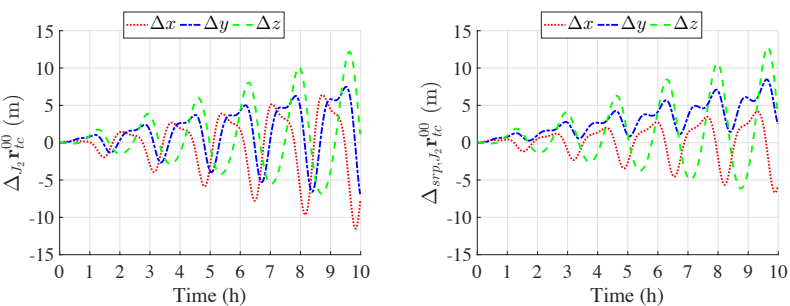

(c) $\Delta_{J_{2}} \boldsymbol{r}_{t c}^{00}={ }_{f, J_{2}} \boldsymbol{r}_{t c}^{00}-{ }_{f} \boldsymbol{r}_{t c}^{00}$

(d) $\Delta_{s r p, J_{2}} \boldsymbol{r}_{t c}^{00}={ }_{c, J_{2}} \boldsymbol{r}_{t c}^{00}-$ ${ }_{f} \boldsymbol{r}_{t c}^{00}$

Fig. 9 Analyses of both dynamic coupling and $J_{2}$ perturbation effects on the relative translational motion.

\section{Conclusions}

This paper proposes a novel six-degrees-of-freedom coupled relative motion model without the attitude synchronization and the Keplerian reference orbit assumptions. This strength makes the proposed model effective to simultaneously analyze, under the influence of orbital perturbations, both the kinematic and dynamic coupling effects on the relative translational motion between arbitrary feature points, as well as to compare results with the perturbation effect. All these effects are illustrated by the numerical propagation of a close SFF mission with different simulation conditions.

It has been demonstrated that the strength of the kinematic coupling effect depends on the magnitudes of the chaser angular velocity and the relative angular velocity. The kinematic coupling effect is always present, even in short operation times, but bounded. On the contrary, the dynamic coupling effect induced by the solar radiation pressure and the perturbation effect in- 
duced by $J_{2}$ are not bounded. They are less dominant in the initial propagation phase, but increase gradually over time and become more significant. Therefore, for a space mission in short operation times, such as in orbital rendezvous and docking, the kinematic coupling effect is dominant, while the dynamic coupling effect and $J_{2}$ perturbation effect is dominant for long-time spacecraft formation flying missions.

Acknowledgements J.C. thanks the support of the Chinese Scholarship Council. The authors also thank useful comments from the referees.

Funding: This study was funded by the Ministerio de Economia y Competividad (grant numbers PGC2018-100928-B-100, MTM2016-80117-P), the Catalan government (grant numbers 2017SGR-1049, 2017SGR-1374) and the National Natural Science Foundation of China (grant number 11572248).

\section{Conflict of interest}

The authors declare that they have no conflict of interest.

\section{References}

1. Yamada, K., Kimura, M., Shima, T., Yoshikawa, S., New state transition matrix for formation flying in $J_{2}$-perturbed elliptic orbits, Journal of guidance, control, and dynamics, 35 (2), 536-547, 2012.

2. Wang, J., Sun, Z., 6-DOF robust adaptive terminal sliding mode control for spacecraft formation flying, Acta astronautica 73, 76-87, 2012.

3. Lee, D., Vukovich, G. Robust adaptive terminal sliding mode control on SE (3) for autonomous spacecraft rendezvous and docking, Nonlinear dynamics, 83 (4), 2263$2279,2016$.

4. Xia, K., Huo, W., Robust adaptive backstepping neural networks control for spacecraft rendezvous and docking with uncertainties, Nonlinear dynamics, 84 (3), 1683-1695, 2016.

5. Segal, S., Gurfil, P., Effect of kinematic rotationtranslation coupling on relative spacecraft translational dynamics, Journal of guidance, control, and dynamics 32 (3), 1045-1050, 2009.

6. Lee, D., Vukovich, G., Kinematically coupled spacecraft relative motion without attitude synchronization assumption, Aerospace science and technology, 45, 316-323, 2015.

7. Clohessy, W., Terminal guidance system for satellite rendezvous, Journal of the aerospace sciences, 27 (9), 653-658, 1960.

8. Tschauner, J., Hempel, P., Rendezvous zu einem in elliptischer bahn umlaufenden ziel, Acta astronautica, 11 (2), 104-109, 1965

9. Yamanaka, K., Ankersen, F., New state transition matrix for relative motion on an arbitrary elliptical orbit, Journal of guidance, control, and dynamics, 25 (1), 60-66, 2002.
10. Ren, Y., Masdemont, J.J., Marcote, M., Gómez, G., Computation of Analytical Solutions of the Relative Motion about a Keplerian Elliptic Orbit, Acta Astronautica, 81, 186-199, 2012.

11. Dang, Z., New state transition matrix for relative motion on an arbitrary keplerian orbit, Journal of guidance, control, and dynamics, 40 (11), 2917-2927, 2017.

12. Chen, W., Jing, W., Dynamics equations of relative motion around an oblate earth with air drag, Journal of aerospace engineering, 25 (1), 21-31, 2012.

13. Kasdin, N. J., Gurfil, P., Kolemen, E., Canonical modelling of relative spacecraft motion via epicyclic orbital elements, Celestial mechanics and dynamical astronomy, 92 (4), 337-370, 2005.

14. Lee, D., Kolemen, E., Spacecraft coupled tracking maneuver using sliding mode control with input saturation, Journal of Aerospace Engineering, 28 (5), 2015.

15. Wang, J., Liang, H., Sun, Z., Zhang, S., Liu, M., Finitetime control for spacecraft formation with dual-numberbased description, Journal of guidance, control, and dynamics 35 (3), 950-962, 2012.

16. Gui, H., Vukovich, G., Dual-quaternion-based adaptive motion tracking of spacecraft with reduced control effort, Nonlinear Dynamics, 83 (1), 597-614, 2016.

17. Filipe, N., Tsiotras, P., Adaptive position and attitudetracking controller for satellite proximity operations using dual quaternions, Journal of guidance, control, and dynamics 38 (4), 566-577, 2014.

18. Filipe, N., Tsiotras, P., Simultaneous position and attitude control without linear and angular velocity feedback using dual quaternions, 2013 American control conference, IEEE, 4808-4813,2013.

19. Tsiotras, P., Valverde, A., Dual Quaternions as a Tool for Modeling, Control, and Estimation for Spacecraft Robotic Servicing Missions, The Journal of the Astronautical Sciences, 67 (2), 595-629, 2020.

20. Aspragathos, N. A., Dimitros, J. K., A comparative study of three methods for robot kinematics, IEEE Transactions on Systems, Man, and Cybernetics, Part B (Cybernetics), 28 (2), 135-145, 1998.

21. Wang, X., Zhu, H., On the comparisons of unit dual quaternion and homogeneous transformation matrix, Advances in Applied Clifford Algebras, 24 (1), 213-229, 2014.

22. Wang, Y., Xu S., On the nonlinear stability of relative equilibria of the full spacecraft dynamics around an asteroid, Nonlinear dynamics, 78 (1), 1-13, 2014.

23. Gao, C., Yuan, J., Zhang, J., Guo, L., Propellant-efficient station-keeping using a hybrid sail in the Earth-Moon system, Nonlinear dynamics, 95 (2), 1323-1346, 2019.

24. Gong, S., Baoyin, H., Li, J., Coupled attitude-orbit dynamics and control for displaced solar orbits, Acta astronautica 65 (5-6), 730-737, 2009.

25. Kumar, B. S., Ng, A., Yoshihara, K., De Ruiter, A., Differential drag as a means of spacecraft formation control, IEEE transactions on aerospace and electronic systems, 47 (2), 1125-1135, 2011.

26. Junkins, J. L., Schaub, H., Analytical mechanics of space systems, Second edition, AIAA education, 2003.

27. Montenbruck, O., Gill, E., Satellite orbits: models, methods, and applications, Chapter 3, Springer science and business media, 2012.

28. Shuster, M. D., A survey of attitude representations, Navigation 8 (9), 439-517, 1993.

29. Wu, Y., Hu, X., Hu, D., Li, T., Lian, J., Strapdown inertial navigation system algorithms based on dual quaternions, IEEE transactions on aerospace and electronic systems 41 (1), 110-132, 2005. 
30. Clifford,: A preliminary sketch of biquaternions, Proceedings of the London mathematical society, s1-4 (1), 381-395, 1873.

31. Han, D. P., Wei, Q., Li, Z. X., Kinematic control of free rigid bodies using dual quaternions, International journal of automation and computing, 5 (3), 319-324, 2008.

32. Hamilton, R. W., On quaternions or on a new system of imaginaries in algebra, The London, Edinburgh, and Dublin philosophical magazine and journal of science (1844-1850).

33. Evans, D. J., On the representatation of orientation space, Molecular physics, 34 (2), 317-325, 1977.

34. Bhat, S. P., Bernstein, D. S., A topological obstruction to continuous global stabilization of rotational motion and the unwinding phenomenon, Systems and control letters, 39 (1), 63-70, 2000.

35. Valverde, A., Tsiotras, P., Dual Quaternion Framework for Modeling of Spacecraft-Mounted Multibody Robotic Systems, Frontiers in Robotics and AI, 5, 128, 2018.

36. Valverde, A., Dynamic modeling and control of spacecraft robotic systems using dual quaternions, Ph.D. dissertation, Dept. Aeros., Georgia Inst. Technol., Georgia, GA, USA, 2018.

37. Brodsky, V., Shoham, M., Dual numbers representation of rigid body dynamics, Mechanism and machine theory, $34(5), 693-718,1999$.

38. Gurfil, P., Relative motion between elliptic orbits: Generalized boundedness conditions and optimal formationkeeping, Journal of guidance control and dynamics, 28 (4), 761$767,2012$. 